\title{
Capillary angiogenesis and degeneration in bovine ovarian antral follicles
}

\author{
J. Y. Jiang ${ }^{1,2}$, G. Macchiarelli ${ }^{3}$, B. K. Tsang ${ }^{2}$ and E. Sato ${ }^{1}$ \\ ${ }^{1}$ Laboratory of Animal Reproduction, Graduate School of Agricultural Science, Tohoku University, Sendai \\ 981-8555, Japan; ${ }^{2}$ Reproductive Biology Unit, Department of Obstetrics \& Gynecology and Cellular \& \\ Molecular Medicine, University of Ottawa, Ontario, Canada, K1Y 4E9; Hormones, Growth and \\ Development Unit, Ottawa Health Research Institute, The Ottawa Hospital (Civic Campus) Ottawa, \\ Ontario, Canada, K1Y 4E9; and ${ }^{3}$ Department of Experimental Medicine, Faculty of Medicine, University \\ of L'Aquila, Via Vetoio, Coppito 2, L'Aquila 67100, Italy
}

\begin{abstract}
Angiogenesis and capillary degeneration are both evident during ovarian follicle growth. However, the characteristics and distribution of thecal capillary proliferative and degenerative structures have not been fully defined. Indeed, the role of thecal microvasculature changes in follicular atresia is still a matter of debate. The present study examined the distribution of thecal capillary changes occurring during follicular growth and related the changes to capillary morphology (by scanning electron microscopy, SEM, on bovine ovarian corrosion casts) with the incidence of capillary apoptosis (TdT-mediated dUTP nick end-labelling, TUNEL) and follicular status (as confirmed by follicular fluid steroid concentrations). SEM demonstrated well-perfused vascular plexuses of small to large antral follicles with structural and functional changes to capillaries. Angiogenesis was evident mainly in the apical part of the inner capillary layer of medium follicles and the
\end{abstract}

middle or basal part of the inner capillary layer of dominant follicles that exhibited high oestradiol:progesterone ratios. Degenerative capillaries were observed mainly in the outer vascular layers of small follicles, and in the inner and outer vascular layers of medium antral follicles. Although apoptotic structures were present only in the outer capillaries of the theca interna of morphologically healthy antral follicles, atretic follicles showed apoptotic structures in both the outer and inner thecal capillary layers. These results show that angiogenesis increases during bovine follicular growth and occurs unevenly in different inner theca regions of the follicles. The differential angiogenic and degenerative response of theca interna capillaries may reflect differences in the microenvironment of the follicles, which in turn determine the fate of the follicles (continued growth versus atresia).

\section{Introduction}

During the prepubertal period and reproductive cycle, the ovarian vasculature changes in size and morphology. These modifications, both functional and structural, mainly occur in the ovarian microvasculature supplying the luteo-follicular complex and appear to play important roles in folliculogenesis, ovarian hormone production and ovulation (Bjersing and Cajander, 1974; Cherney et al., 1975; Ellinwood et al., 1978; Espey, 1980; Meyer, 1991; Nottola et al., 1997). Indeed, the development of a healthy vascular supply is a fundamental requirement for organ growth and differentiation during embryogenesis, wound healing and reproductive functions (Ferrara et al., 1996; Macchiarelli, 2000). Although an impairment of follicular blood vascular supply has been observed during atresia (Greenwald and Terranova, 1988), it is still a matter of debate

Email: jjiang@ohri.ca whether follicular ischaemia is a cause or consequence of atresia. In addition, the distribution of capillary degenerative patterns in follicular microvascular architecture and its physiological significance have not been fully elucidated.

Scanning electron microscopy (SEM) studies of ovarian follicle corrosion casts allow the visualization of the dynamic microvascular architectural changes during follicular development, ovulation and atresia (Kardon and Kessel, 1979; Kanzaki et al., 1982; Takada et al., 1987; Murakami et al., 1988; Macchiarelli et al., 1991, 1992, 1993, 1995, 1998; Forsman and McCormack, 1992; Shimoda et al., 1993; Yamada et al., 1994; Nottola et al., 1997; Macchiarelli, 2000). In addition, SEM of vascular corrosion casting offers a quasithree-dimensional, high resolution imaging approach for detailed morphological spatial analysis of microvascular systems (Murakami, 1971; Lametschwandtner et al., 1990; Miodonski and Litwin, 1999). SEM studies have facilitated the characterization of capillary proliferation 
and regression in the microvasculature of growing and atretic rabbit ovarian follicles (Macchiarelli et al., 1993) and a detailed analysis of bovine thecal capillaries with minimal artefacts (Ginther and Del Campo, 1974; Lamond and Drost, 1974; Yamada et al., 1994, 1995).

Recent studies in the pupillary membrane and retina demonstrated that endothelial cell apoptosis is responsible for the regression of capillaries (Lang and Bishop, 1993; Lang et al., 1994; Meeson et al., 1996; Meeson et al., 1999; Yamada et al., 1999). However, to the authors' knowledge, there have been no reports of application of the TdT-mediated dUTP nick end-labelling (TUNEL) method to characterize capillary regression in ovarian follicles. The present study used SEM on vascular corrosion cast to describe the distribution of proliferative (angiogenic) and degenerative capillary structures in the thecal microvasculature of bovine follicles, and examine the nature of the capillary degenerative aspects in the context of follicular growth and atresia.

\section{Materials and Methods}

\section{Collection of bovine ovaries and follicular fluid}

Ovaries ( $n=32)$ of Japanese black cattle collected at a local abattoir were placed in heparinized saline solution and transported to the laboratory. The animals were considered to be in the follicular phase of the oestrous cycle (21 ovaries) if corpora lutea were absent, and to be in the luteal phase (11 ovaries) if corpora lutea were present. The diameter of follicles was measured and the position of follicles recorded. Follicular fluids were then aspirated with a $28 \mathrm{G} 1 / 2$ needle and stored at $-40^{\circ} \mathrm{C}$ until assay.

\section{SEM of vascular corrosion casts}

Ovaries were prepared for corrosion casts, as reported by Jiang et al. (2002) and Shimizu et al. (2002), regardless of follicular fluid collection. Briefly, the ovaries were perfused with heparinized saline solution and subsequently with a solution of Mercox (Okenshoji, Tokyo) through the ovarian artery. Cast ovaries were then polymerized in hot water at $60^{\circ} \mathrm{C}$ for $2 \mathrm{~h}$, corroded in $10 \% \mathrm{NaOH}$ at $60^{\circ} \mathrm{C}$, washed overnight in warm running tap water $\left(50^{\circ} \mathrm{C}\right)$ and dried in a hot oven. Dried samples were glued onto aluminium stubs and coated with platinum. An SEM (Hitachi S-4200, Tokyo) was used at low voltage and deep working distance. The samples were frozen and then cracked with a cooled razor blade to observe the inner wall of the follicular microvasculature. These cracked samples were dried and mounted in stubs before further coating and observation.

Vessels were classified according to their diameter and the shape of endothelial cell nuclei (Macchiarelli et al., 1991, 1993, 1995, 1998). Budding, sprouting and splitting (by transcapillary pillars or posts of extracellular matrix) of capillaries from pre-existing blood vessels were considered as proliferative (angiogenic) features (Macchiarelli et al., 1991, 1995; Augustin et al., 1995; Yamada et al., 1995; Risau, 1997; Macchiarelli, 2000). Incompletely filled or thinned capillaries were considered degenerative (Macchiarelli et al., 1993; Meeson et al., 1999; Yamada et al., 1999; Macchiarelli, 2000). The numbers of angiogenic and degenerative structures in a defined area $\left(0.0378 \mathrm{~mm}^{2}\right)$ in different regions of the follicular plexus were counted.

\section{TUNEL staining}

Ovaries $(n=3)$ from three cows at the follicular phase were cut in half and placed in $10 \%$ neutral buffered formalin immediately after collection. The ovaries were transported to the laboratory, dissected into pieces of about $1 \mathrm{~cm}^{3}$ and stored in formalin solution for a further $48 \mathrm{~h}$. Fixed samples were dehydrated in an ethanol step gradient and embedded in paraffin wax. Serial sections cut at a thickness of $5 \mu \mathrm{m}$ were subjected to TUNEL staining using a commercial kit and according to the manufacturer's protocol (ApopTag ${ }^{\circledR}$ Plus Peroxidase In Situ Apoptosis Detection Kit, cat. no. S7101; Intergen Company, NY). Briefly, after de-paraffination and rehydration, the sections were incubated with proteinase $\mathrm{K}(15 \mathrm{~min})$, washed twice with deionized water, and incubated with $\mathrm{H}_{2} \mathrm{O}_{2}(3 \%, 5 \mathrm{~min}$; to remove endogenous peroxidase activity) and TdT equilibration buffer ( $5 \mathrm{~min}$ ). The sections were then incubated in fresh TdT enzyme (60 min; TUNEL reaction), rinsed three times with PBS without $\mathrm{Mg}^{2+}(\mathrm{PBS}(-))$, and incubated with antidigoxigenin peroxidase conjugate $\left(30 \mathrm{~min}\right.$ at $26^{\circ} \mathrm{C} \pm$ $\left.1^{\circ} \mathrm{C}\right)$. After four washes with PBS(-), the sections were incubated with diamino benzidine and mounted with Entellan (Merck, Darmstadt).

Atretic follicles were evaluated according to the criteria reported by Guthrie et al. (1993, 1995) and Garrett and Guthrie (1996). Follicles with more than $10 \%$ labelled granulosa cells were classified as atretic. The numbers of capillaries with TUNEL-positive and -negative signals in defined areas in different regions (inner, middle and outer layers) were counted in three follicles (from three cows) at each follicular status and size. The areas $(w \times h)$ of inner layer counted in small and medium healthy follicles and medium atretic follicles were $214 \times 10 \mu \mathrm{m}^{2}$, and those of both middle and outer layers in small healthy follicles and middle follicles (healthy and atretic) were $214 \times 45 \mu \mathrm{m}^{2}$, and $214 \times$ $70 \mu \mathrm{m}^{2}$, respectively.

\section{Determination of steroid concentrations}

After centrifugation ( $900 \mathrm{~g}$ for $10 \mathrm{~min}$ at $4^{\circ} \mathrm{C}$ ) to remove whole cells and cellular debris, the follicular fluids were diluted 100 times with DELFIA ${ }^{\circledR}$ diluent I 
and oestradiol and progesterone concentrations were determined with kits (DELFIA ${ }^{\circledR}$ oestradiol reagents R056101J and DELFIA ${ }^{\circledR}$ progesterone reagents R066-101J, respectively; Pharmacia Biotech KK, Tokyo), as reported by Jiang et al. (2000a, 2001a). The limits of sensitivity were $13.6 \mathrm{pg} \mathrm{ml}^{-1}$ and $0.25 \mathrm{ng} \mathrm{ml}^{-1}$ for oestradiol and progesterone assays, respectively. The interassay and intra-assay coefficients of variation were $4.6 \%$ and $5.7 \%$ (oestradiol assay) and $8.1 \%$ and $4.2 \%$ (progesterone assay), respectively. Follicles were considered healthy when the concentration ratio of oestradiol:progesterone was $>1.0$, and as atretic when the ratio was $<1.0$, as defined by Roth et al. (2001).

\section{Statistical analysis}

A Student's $t$ test was used to compare differences in the numbers of angiogenic and degenerative structures in defined areas of the inner layer plexus in dominant follicles at the follicular phase. ANOVA and Fisher's PLSD multiple-range tests were used for other comparisons. Differences were considered significant at $P<0.05$.

\section{Results}

\section{SEM of vascular corrosion casts}

SEM permitted the identification of numerous plexuses, mainly ovoid and of different size, that appeared well-perfused by the casting medium. About 53\% (62 of 118) of the plexuses examined were small $(<3 \mathrm{~mm}$ in diameter), $42 \%$ (49 of 118) were of medium size (3-7 mm in diameter) and $6 \%$ (7 of 118) were large (10-15 $\mathrm{mm}$ in diameter). Medium and large plexuses were mainly supplied by individual spiral arteries and drained by several enlarged veins that emptied in the cortical vessels (Fig. 1). The smallest plexuses showed a vascular supply and drainage mainly shared with the very scant vascularization of the interstitium. Cracked medium and large plexuses showed a multilayered wall. The inner layer was composed of a dense capillary network supplied by arterioles in healthy follicles and drained by venules distributed in the outer layer. The outer layer also contained numerous capillaries. Most of the plexuses presented both proliferative (angiogenic) and degenerative aspects of the capillaries. However, distribution of these structures varied greatly, according to the plexus size (small, medium and large), the follicle status (healthy versus atretic), the capillary layers (inner or outer layer), and the inner capillary layer regions (basal, equatorial and apical) of an individual plexus.

Small plexuses (Fig. 2) were more numerous (53\%, 62 of 118) than medium and large plexuses and presented two different patterns (Small A and Small B) of distribution of capillary proliferative and degenerative characteristics. In Small A (37\%, 23 of 62; Fig. 2a,b), the outer capillary network (Fig. 2a) was derived from normal capillaries, whereas the inner capillary network (Fig. 2b) presented numerous budding capillaries. Small B (63\%, 39 of 62; Fig. 2c,d) showed degenerative structures in the capillaries of the outer layer (Fig. 2c) represented by thin or incompletely filled capillaries. The inner capillary network of these small follicles showed some signs of angiogenesis such as capillary budding (37\%, 23 of 62; Fig. 2d) and capillary degeneration (26\%, 16 of 62$)$.

Medium plexuses $(42 \%, 49$ of 118 , Fig. 3) showed two types of pattern (Medium A and Medium B) of distribution of proliferative and degenerative capillaries. For Medium A plexuses $(51 \%, 25$ of 49, Fig. 3a-c), the outer capillary network (Fig. 3a) presented both thinned capillaries in certain regions (data not shown) and capillary budding or sprouting in other regions (Fig. 3a, inset). The inner layer showed mainly normal capillaries with few proliferative characteristics in the basal region (Fig. 3b) and numerous angiogenic features (sprouting and splitting) in the apical region (Fig. 3c). Medium B plexuses (49\%, 24 of 49; Fig. 3d,e) presented degenerative characteristics (thin or incompletely filled capillaries) distributed in both the outer (Fig. 3d) and inner capillary network (Fig. 3e).

Large plexuses (>10 mm in diameter; Figs 1 and 4$)$ were observed less frequently $(6 \%, 7$ of 118$)$. Dominant follicles at the follicular phase of the oestrous cycle $(100 \%$, three of three from three cows) showed a rich outer layer with normal and mostly dilated capillaries (Fig. 4a), and an inner layer of dilated capillaries with different angiogenic structures in different locations. Differences were observed in the density and diameter of capillaries in neighbouring areas (Fig. 4b-d) between the equatorial and the basal region of the inner layer network. Inner capillaries in the apical region (Fig. 4e) showed extensive budding and splitting, and those in the equatorial area (Fig. 4f) exhibited mostly angiogenesis with sprouting, whereas those in the basal region (Fig. 4g) showed normal but significantly fewer angiogenic structures $(P<0.05$; Table 1$)$. All plexuses of non-dominant follicles at the follicular phase of the oestrous cycle ( $100 \%$, two of two) and dominant follicles at the corpus luteum phase $(100 \%$, two of two) showed many large avascular areas and few capillaries with angiogenic structures in the inner layer (Fig. 5).

\section{TUNEL staining}

Healthy small antral follicles (Fig. $6 \mathrm{a}, \mathrm{d} ;<3 \mathrm{~mm}$ in diameter) were characterized by the presence of a few thecal capillaries in the inner layer and the absence of apoptosis (Table 2). Healthy medium-sized follicles (Fig. 6b,e; 3-7 mm) showed significantly more capillaries in the inner layer compared with healthy small antral follicles, with a few apoptotic cells localized in the outer layer of the theca interna. Atretic medium antral 


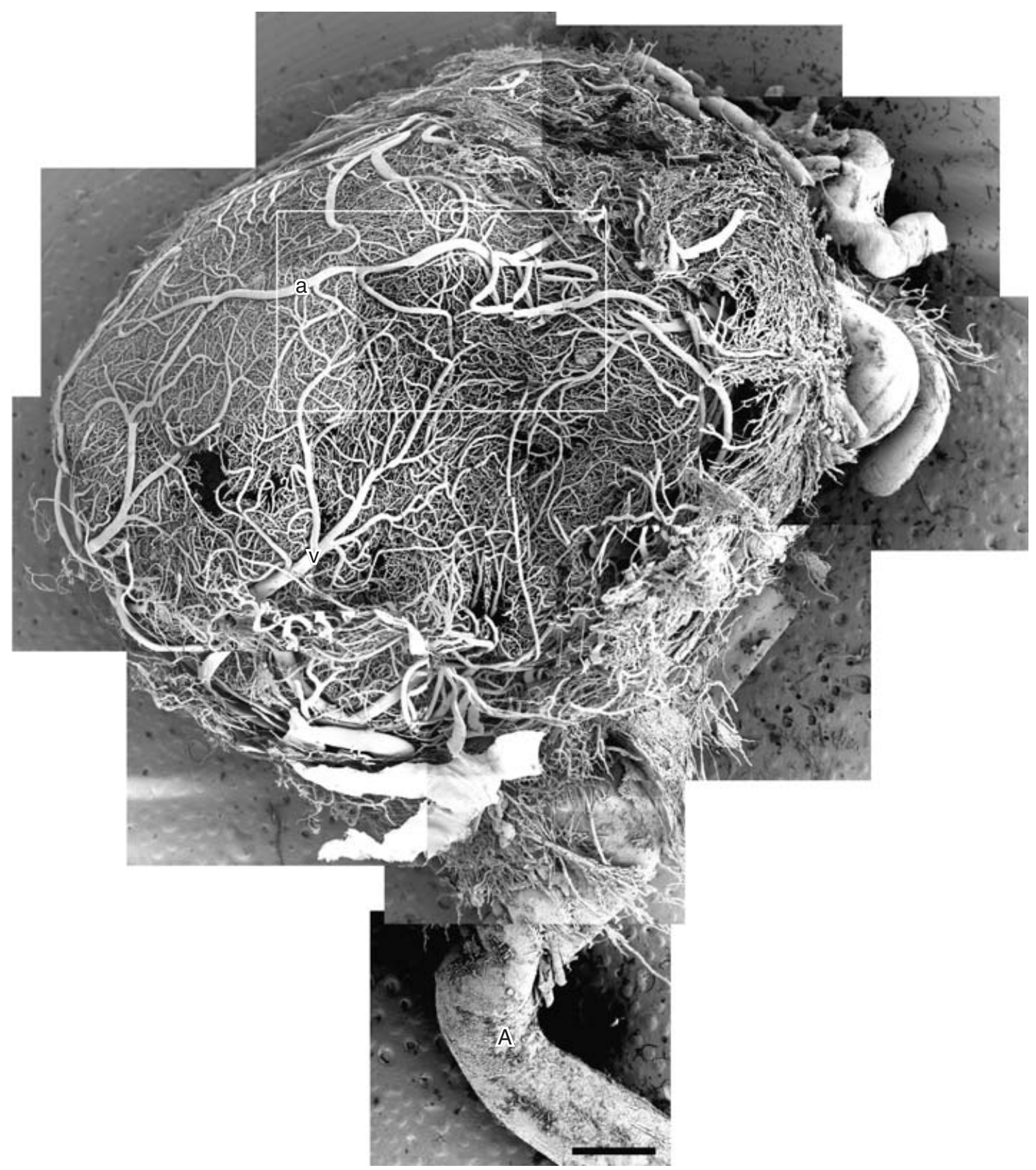

Fig. 1. Outer layer capillaries of a bovine large antral follicle (12 $\mathrm{mm}$ in diameter). a, arteriole; v, venule; $A$, artery. Scale bar represents $1.38 \mathrm{~mm}$.

follicles (Fig. 6c,f) exhibited significantly more apoptotic cells in both middle and outer thecal capillaries, but a marked decrease in the number of healthy capillaries in the inner layer compared with that of healthy medium follicles.

\section{Steroid concentrations in follicular fluid}

All dominant follicles $(100 \%$, three of three) collected from ovaries of three cows at the follicular phase of the oestrous cycle had well-developed capillaries and active angiogenic structures in the inner layer of the vascular plexus (Figs 1 and 4) and significantly higher oestradiol:progesterone ratios in the follicular fluid (Table 3). All non-dominant large follicles (100\%, two of two) at the follicular phase of the oestrous cycle, and $67 \%$ (two of three) of dominant follicles and $63 \%$ (five of eight) of non-dominant follicles at the luteal phase had significantly lower oestradiol:progesterone ratios. 
Table 1. Number of angiogenic or degenerative structures in different regions $\left(0.0378 \mathrm{~mm}^{2}\right)$ of the inner plexus of dominant follicles in cows

\begin{tabular}{lccc}
\hline & \multicolumn{3}{c}{ Region } \\
\cline { 2 - 4 } & Apical & Equatorial & Basal \\
\hline Number of angiogenic structures & $3.7 \pm 0.3^{\mathrm{a}}$ & $9.5 \pm 0.5^{\mathrm{b}}$ & $1.4 \pm 0.3^{\mathrm{c}}$ \\
Number of degenerative structures & $0.3 \pm 0.3^{*}$ & $0 \pm 0^{*}$ & $0.4 \pm 0.3^{*}$ \\
\hline
\end{tabular}

Values represent mean $\pm \operatorname{SEM}(n=3$ follicles from three cows examined).

abc Values with different superscripts are significantly different $(P<0.05)$.

*Significantly different from value within the same column $(P<0.05)$.
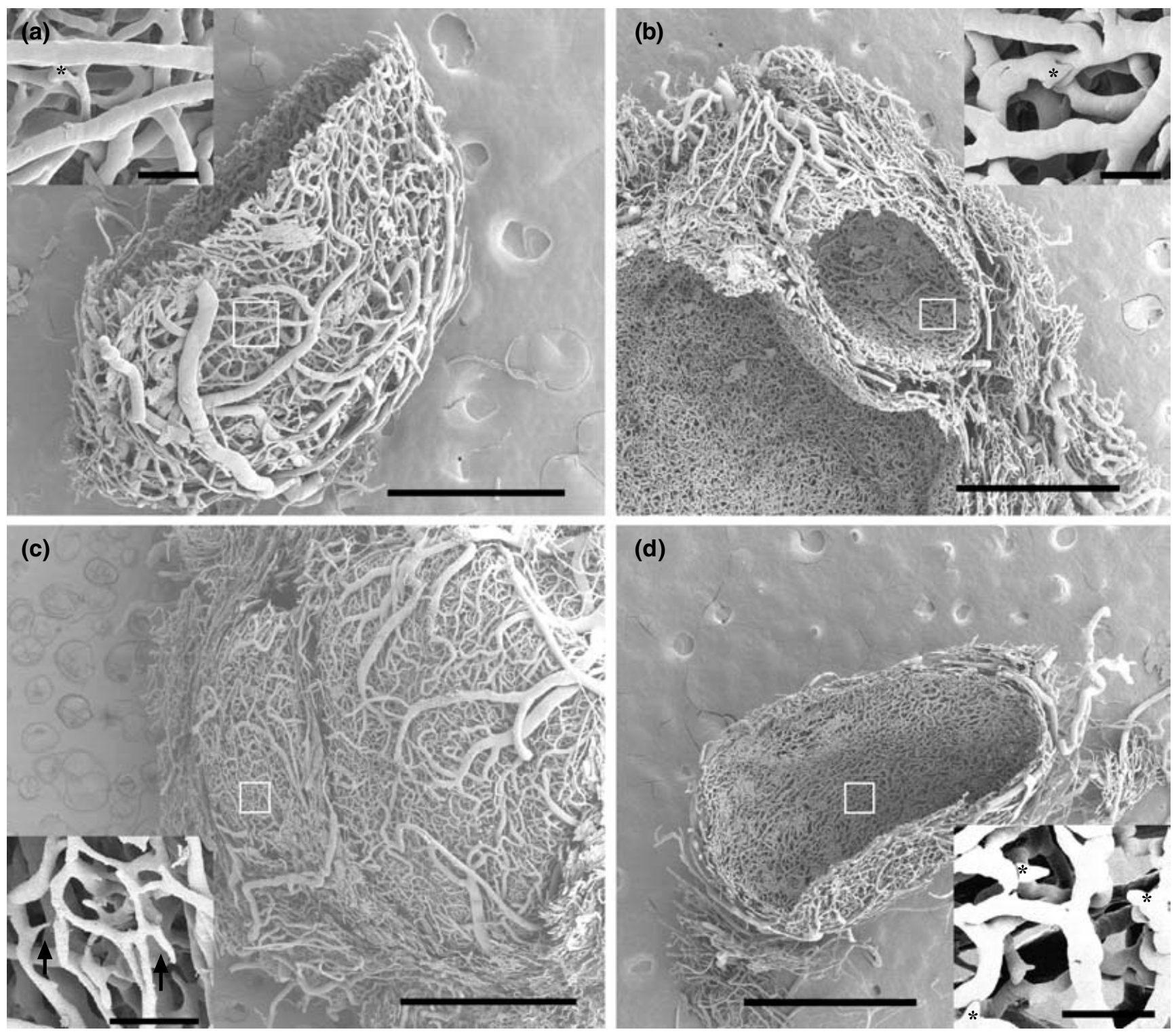

Fig. 2. $(a, c)$ Outer and $(b, d)$ inner layer of capillaries in bovine small antral follicles. SEM identified two different patterns of capillary proliferative and degenerative characteristics: Small A $(1.2 \mathrm{~mm}$ in diameter) and Small B (2.0 mm in diameter). Insets show higher magnification views of selected areas in corresponding figures. Budding is indicated by asterisks and degenerative capillaries are indicated by arrows. Scale bars represent (a) $720 \mu \mathrm{m}$; (b-d) $1.38 \mathrm{~mm}$; (insets in a,b) $40 \mu \mathrm{m}$; and (insets in c,d) $120 \mu \mathrm{m}$. 

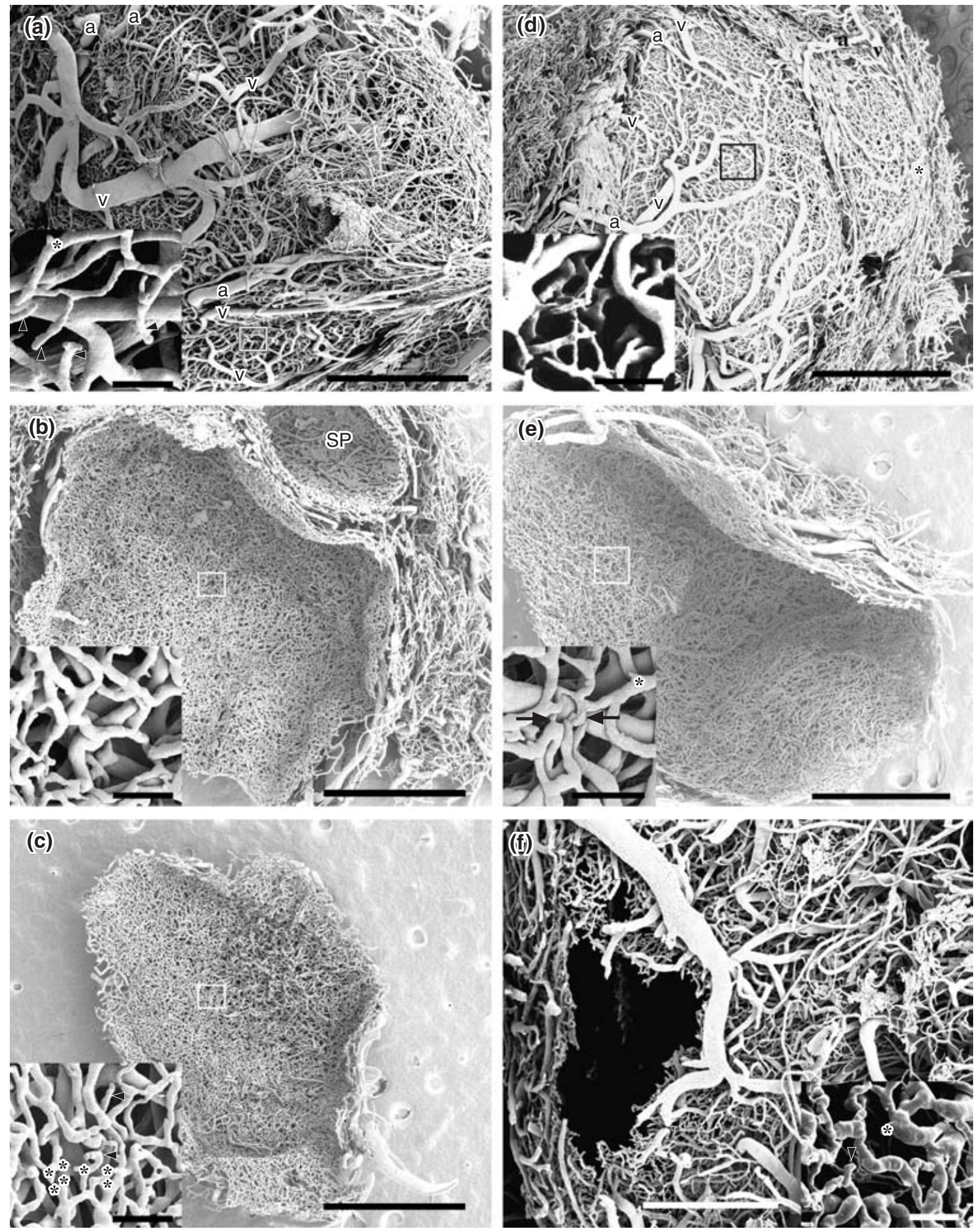

Fig. 3. Capillaries in a bovine medium antral follicle. (a) Outer layer, and (b) basal and (c) apical inner layer capillaries in a medium antral follicle. (d) Outer and (e) inner layer capillaries in another medium antral follicle. SEM identified two different patterns of capillary proliferative and degenerative characteristics: Medium A (a-c; $4.0 \mathrm{~mm}$ in diameter) and Medium B (d,e; $3.7 \mathrm{~mm}$ in diameter). Insets show higher magnification views of selected areas in corresponding figures. (f) A needle opening made by follicular fluid collection before perfusion. The inset shows the inner layer capillaries of the follicular plexus. Budding, sprouting and degenerative capillaries are indicated by asterisks, arrowheads and arrows, respectively. SP, small plexus; a, arteriole; v, venule. Scale bars represent (a-e) $1.38 \mathrm{~mm}$; (f) $1.0 \mathrm{~mm}$; (insets except (f)) $120 \mu \mathrm{m}$; and (inset in (f)) $60 \mu \mathrm{m}$. 

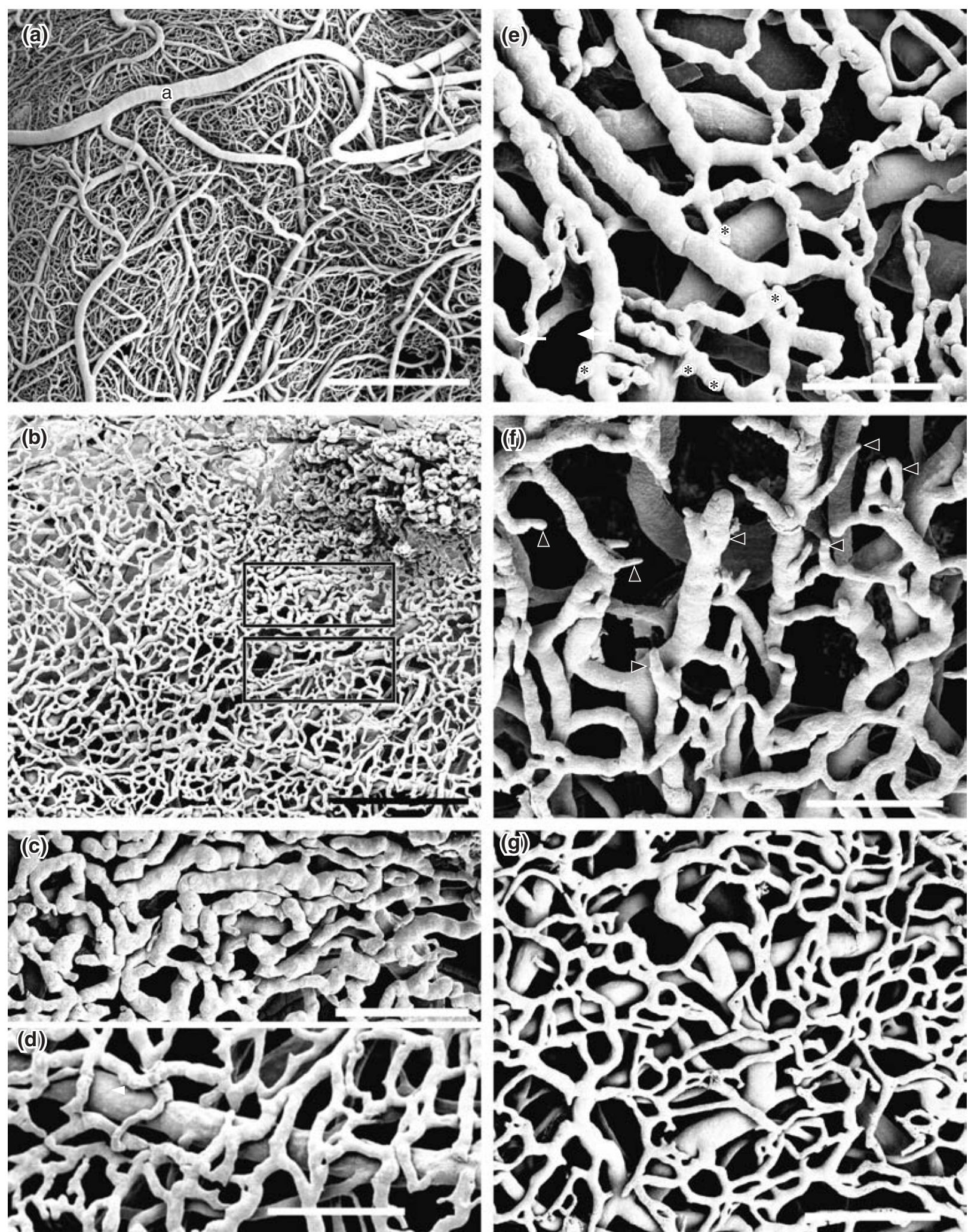

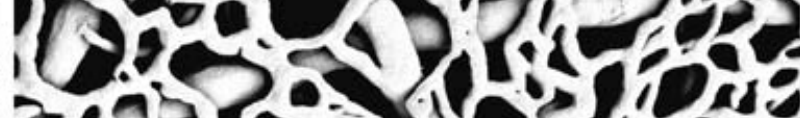

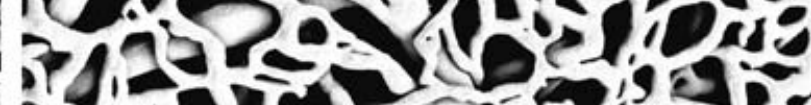

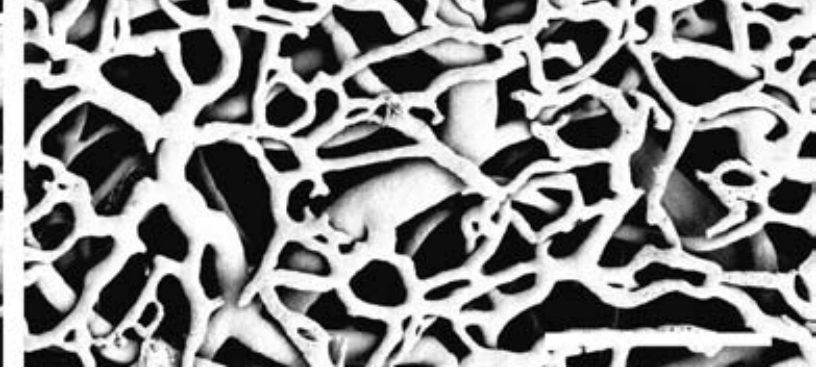

Fig. 4. Capillaries in a bovine large antral follicle. (a) High magnification view of selected area of outer layer capillaries of a large antral follicle. (c,d) High magnification views of neighbouring areas. (b) Area between the equatorial and basal regions of the inner layer capillaries of the large antral follicle (shown in Fig. 1), showing capillaries of different diameter and variable density. (e) Apical, (f) equatorial and (g) basal inner layer capillaries in the large antral follicle (Fig. 1). Different angiogenic characteristics were observed in different locations. Budding and sprouting are indicated by asterisks and arrowheads, respectively. a, arteriole. Scale bars represent (a) $1.38 \mathrm{~mm}$; (b) $400 \mu \mathrm{m}$; (c-f) $120 \mu \mathrm{m}$; and (g) $164 \mu \mathrm{m}$. 
Table 2. Number of bovine capillaries with negative or positive staining of TdT-mediated dUTP nick end-labelling in the theca interna of follicles

\begin{tabular}{|c|c|c|c|c|c|}
\hline \multirow[b]{2}{*}{ Follicle status } & \multirow{2}{*}{$\begin{array}{l}\text { Follicle diameter } \\
\qquad(\mathrm{mm})\end{array}$} & \multirow{2}{*}{$\begin{array}{l}\text { Position in theca } \\
\text { interna }\end{array}$} & \multicolumn{3}{|c|}{ Number of capillaries examined with } \\
\hline & & & Total & Negative signals & Positive signals \\
\hline \multirow[t]{3}{*}{ Healthy } & $<3$ & Inner layer & $0 \pm 0^{a}$ & $0 \pm 0^{a}$ & $0 \pm 0^{\mathrm{a}}$ \\
\hline & & Middle layer & $2.7 \pm 0.7^{\mathrm{b}}$ & $2.7 \pm 0.7^{b}$ & $0 \pm 0^{\mathrm{a}}$ \\
\hline & & Outer layer & $1.7 \pm 0.7^{\mathrm{ab}}$ & $1.7 \pm 0.7^{\mathrm{ab}}$ & $0 \pm 0^{\mathrm{a}}$ \\
\hline \multirow[t]{3}{*}{ Healthy } & $3-7$ & Inner layer & $2.7 \pm 0.3^{b}$ & $2.7 \pm 0.3^{b}$ & $0 \pm 0^{\mathrm{a}}$ \\
\hline & & Middle layer & $3.3 \pm 1.3^{b}$ & $3.3 \pm 1.3^{b}$ & $0 \pm 0^{\mathrm{a}}$ \\
\hline & & Outer layer & $2.3 \pm 0.3^{b}$ & $1.3 \pm 0.3^{\mathrm{a}}$ & $1.0 \pm 0.0^{\mathrm{a}}$ \\
\hline \multirow[t]{3}{*}{ Atretic } & $3-7$ & Inner layer & $0.7 \pm 0.7^{a}$ & $0 \pm 0^{\mathrm{a}}$ & $0.7 \pm 0.7^{a}$ \\
\hline & & Middle layer & $3.7 \pm 0.7^{b}$ & $0 \pm 0^{a}$ & $3.7 \pm 0.7^{b}$ \\
\hline & & Outer layer & $10.0 \pm 1.0^{\mathrm{c}}$ & $1.0 \pm 0.0^{\mathrm{a}}$ & $9.0 \pm 1.0^{c}$ \\
\hline
\end{tabular}

Values represent mean $\pm \operatorname{SEM}(n=3$ follicles from three cows examined).

Values with different superscripts within the same column are significantly different (total, abc $P<0.05$; negative, ab $P<0.05$; positive, abc $P<0.001)$.
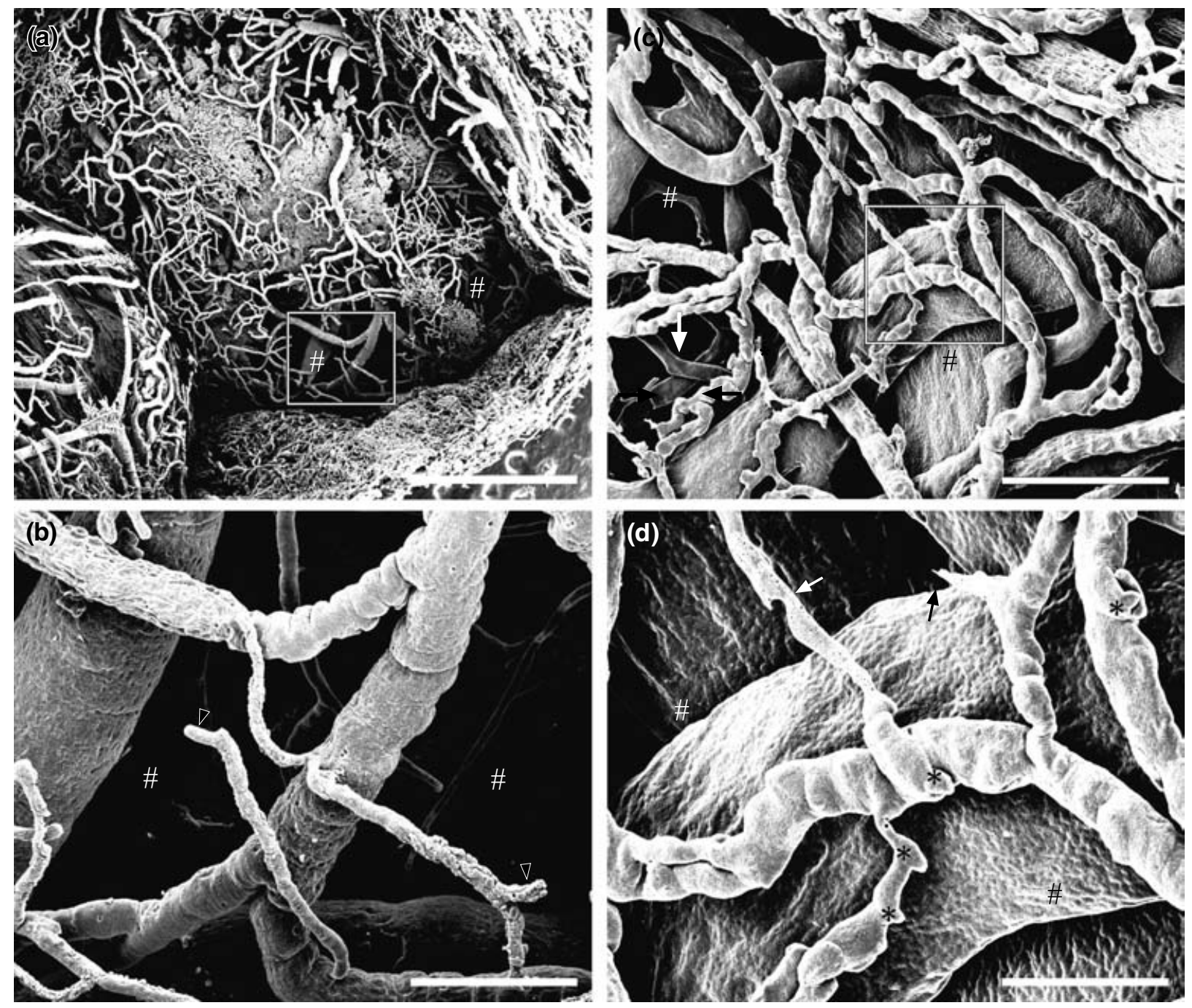

Fig. 5. Inner layer plexus in a bovine atretic antral follicle. $(a, b)$ Medium and (c,d) large antral follicles showing large avascular areas (\#), although some angiogenic structures were also observed. (b,d) Higher magnification views of selected areas in (a) and (c), respectively. Budding, sprouting and degenerative capillaries are indicated by asterisks, arrowheads and arrows, respectively. Scale bars represent (a) $1.38 \mathrm{~mm}$; (b,c) $200 \mu \mathrm{m}$; and (d) $60 \mu \mathrm{m}$. 

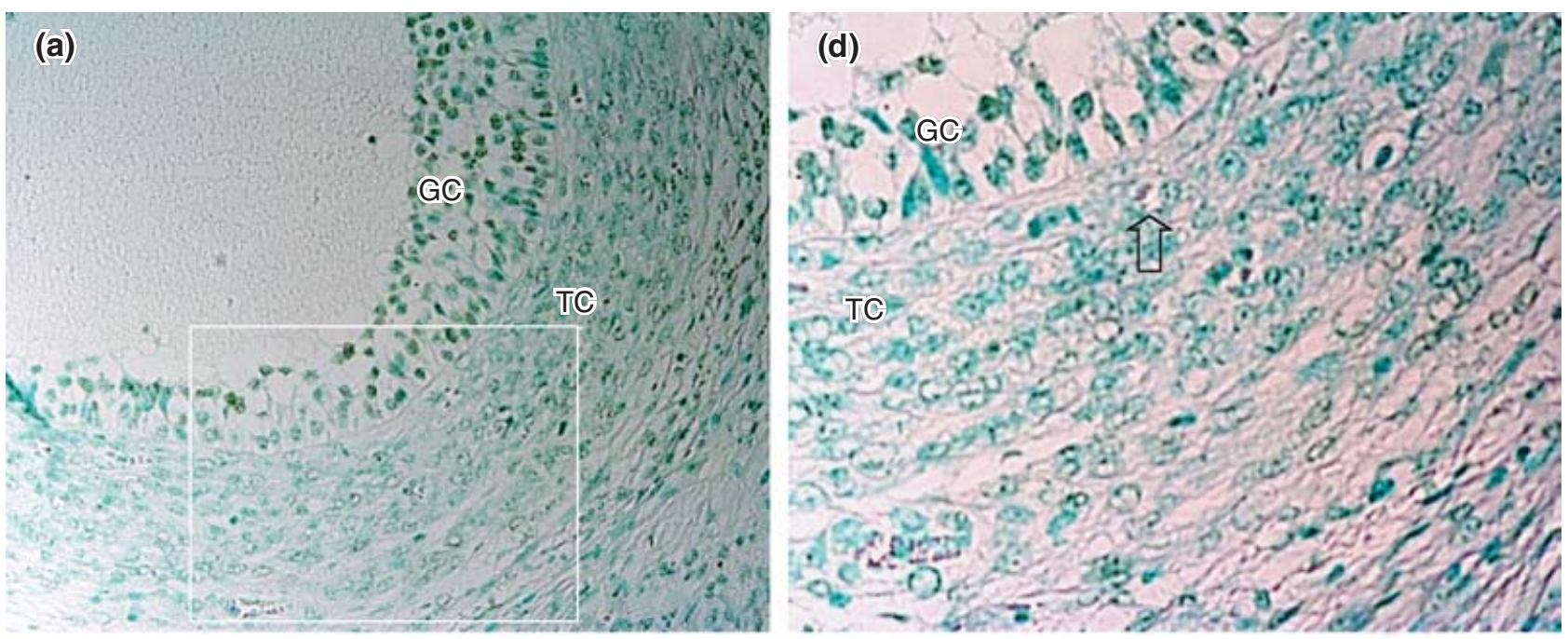

(b)
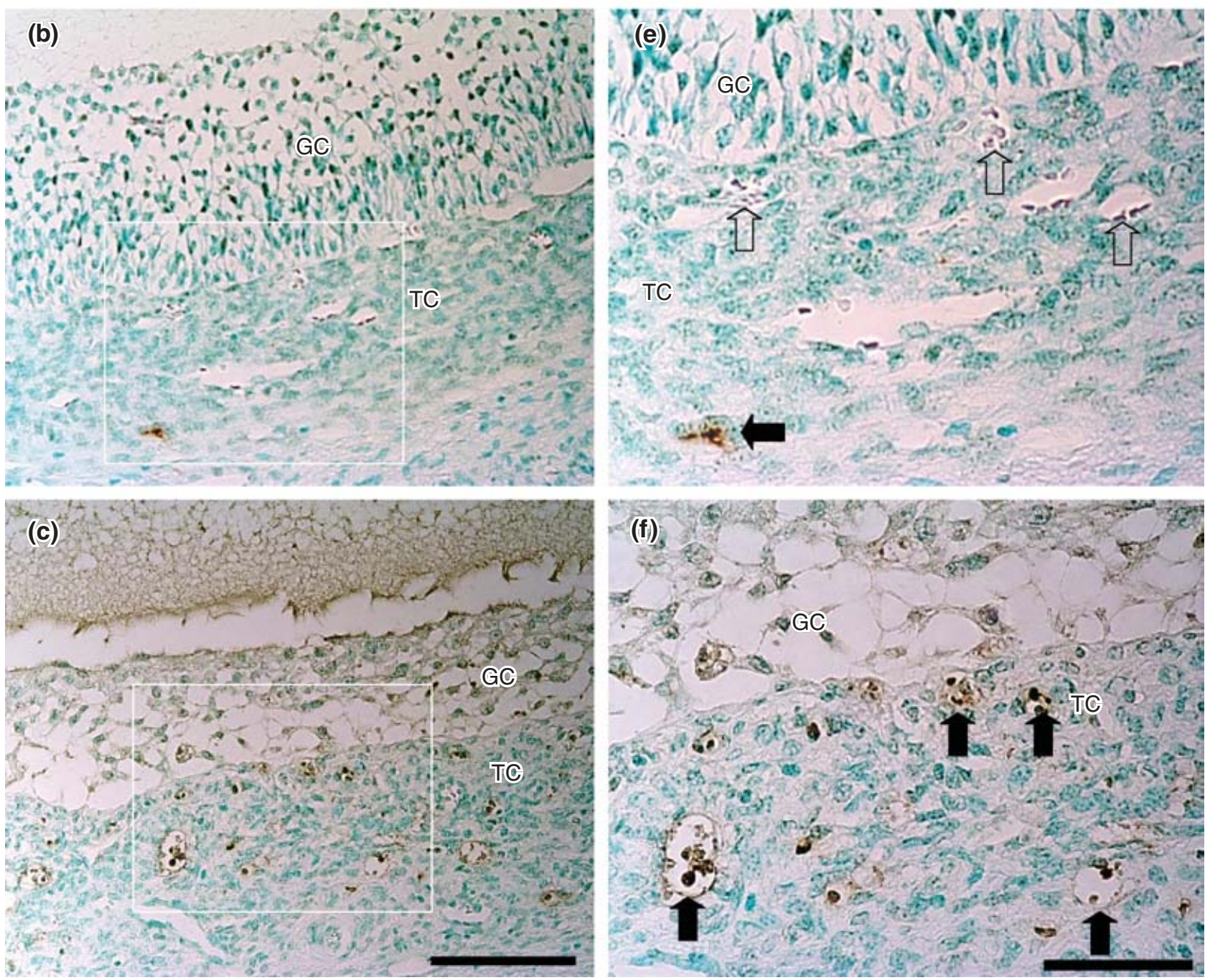

Fig. 6. Sections of bovine healthy antral follicles stained by TdT-mediated dUTP nick end-labelling (TUNEL). (a) Small, (b) medium and (c) atretic medium antral follicles. (d-f) Higher magnification views of selected areas in (a), (b) and (c), respectively. Open and closed arrows indicate healthy and degenerative capillaries, respectively. GC, granulosa cells; TC, theca cells. Scale bars represent (a-c) $100 \mu \mathrm{m}$; and (d-f) $50 \mu \mathrm{m}$. 
Table 3. Follicular fluid concentrations of oestradiol and progesterone in dominant and non-dominant bovine follicles

\begin{tabular}{|c|c|c|c|c|}
\hline & \multicolumn{2}{|c|}{ Follicular phase } & \multicolumn{2}{|c|}{ Luteal phase } \\
\hline & Healthy & Atretic & Healthy & Atretic \\
\hline \multicolumn{5}{|l|}{ Dominant follicles } \\
\hline Number of follicles examined & 3 of 3 & 0 of 3 & 1 of 3 & 2 of 3 \\
\hline Diameter (mm) & $12.3 \pm 1.5^{\mathrm{a}}$ & $\mathrm{N} / \mathrm{A}$ & 10.0 & $10.0,15.0$ \\
\hline Oestradiol $\left(\mathrm{ng} \mathrm{ml}^{-1}\right)$ & $484.0 \pm 26.5^{\mathrm{a}}$ & $\mathrm{N} / \mathrm{A}$ & 509.5 & $0.4,1.6$ \\
\hline Progesterone $\left(\mathrm{ng} \mathrm{ml}^{-1}\right)$ & $29.6 \pm 29.1$ & $\mathrm{~N} / \mathrm{A}$ & 7.6 & $877.8,22.9$ \\
\hline Oestradiol:progesterone ratio & $616.8 \pm 306.5^{a}$ & $\mathrm{~N} / \mathrm{A}$ & 67.0 & $0.0,0.1$ \\
\hline Capillary & $\begin{array}{l}\text { High density and well } \\
\text { developed }\end{array}$ & $\mathrm{N} / \mathrm{A}$ & $\mathrm{N} / \mathrm{A}$ & $\begin{array}{c}\text { Sparse and poorly } \\
\text { developed }\end{array}$ \\
\hline \multicolumn{5}{|l|}{ Non-dominant smaller follicles } \\
\hline Number of follicles examined & 0 of 2 & 2 of 2 & 3 of 8 & 5 of 8 \\
\hline Diameter (mm) & $\mathrm{N} / \mathrm{A}$ & $5.0,12.0$ & $6.7 \pm 0.3^{b}$ & $4.8 \pm 0.2^{b}$ \\
\hline Oestradiol $\left(\mathrm{ng} \mathrm{ml}^{-1}\right)$ & $\mathrm{N} / \mathrm{A}$ & $0.2,27.9$ & $24.0 \pm 5.4^{b}$ & $0.2 \pm 0.1^{b}$ \\
\hline Progesterone (ng ml-1) & $\mathrm{N} / \mathrm{A}$ & $12.5,96.9$ & $0.5 \pm 0.0$ & $0.4 \pm 0.1$ \\
\hline Oestradiol:progesterone ratio & N/A & $0.0,0.3$ & $48.0 \pm 10.7^{b}$ & $0.4 \pm 0.2^{b}$ \\
\hline Capillary & $\mathrm{N} / \mathrm{A}$ & $\begin{array}{c}\text { Sparse and poorly } \\
\text { developed }\end{array}$ & Active angiogenesis & $\begin{array}{c}\text { Sparse and poorly } \\
\text { developed }\end{array}$ \\
\hline
\end{tabular}

Values represent mean \pm SEM; N/A: not available.

Values with different superscripts within the same category are significantly different (diameter, ab $P<0.05$; oestradiol, ab $P<0.0001$; oestradiol:progesterone ratio, ${ }^{\text {ab }} P<0.05$ ).

\section{Discussion}

This study used SEM observations of corrosion cast to demonstrate for the first time in cattle that small healthy ovarian follicles have few capillaries in the inner layer and medium healthy follicles have significantly more capillaries with active angiogenesis. Dominant follicles at the follicular phase of the oestrous cycle have significantly higher oestradiol:progesterone ratios, and well-developed capillaries with active and spatialdependent angiogenesis in the inner layers. Capillaries in medium and large follicles with significantly lower oestradiol:progesterone ratios are sparse and show signs of poor development (for example, thin and incompletely filled capillaries). In addition, distribution and development of capillaries in most plexuses in dominant follicles (at the follicular phase with high oestradiol:progesterone ratios) are more uniform than those of atretic medium and large follicles (with low oestradiol:progesterone ratios). These findings support the concept that follicular development and atresia is accompanied by significant thecal capillary structural and functional changes. Structural changes may be proliferative (angiogenesis) or degenerative in nature; changes that are degenerative accompany follicular atresia (Macchiarelli, 2000).

The microvasculature of ovarian follicles plays important roles in folliculogenesis, ovulation and ovarian hormone production (Yamada et al., 1994). The microvascular architecture of follicles has been studied in rodents, pigs, horses, cows and sheep mainly by SEM of corrosion casts (Kardon and Kessel, 1979; Kanzaki et al., 1982; Kitai et al., 1985; Spanel-Borowski et al., 1987; Murakami et al., 1988; Murdoch and Cavender,1989; Kikuta et al., 1991; Macchiarelli et al., 1991, 1992; Jiang et al., 2002). However, little is known about how microvasculature grows or degenerates. The present study evaluated the occurrence and distribution of degenerative and proliferative structures in the thecal capillaries in order to characterize the vascular changes accompanying follicular development and atresia. SEM of bovine ovarian vascular corrosion casts (the technique of choice for studying spatial distribution of microvessels) was used to identify several types of vascular plexus presenting numerous degenerative or proliferative aspects, morphologically comparable to those reported by Macchiarelli et al. (1993, 2000). The degenerative structures were more abundant in medium plexuses $(49 \%, 24$ of 49$)$ and less abundant in small plexuses $(26 \%, 16$ of 62$)$. In contrast, large plexuses and small to medium plexuses, characterized by the presence of numerous proliferative capillaries, were present in antral follicles independent of the stage of development. Thus, consistent with previous reports (Macchiarelli et al., 1993; Yamada et al., 1999; Macchiarelli, 2000) and supported by the current demonstration of capillary apoptosis and follicles with low oestradiol:progesterone ratios, these degenerative plexuses are likely to be involved in the process of atresia.

Results from the present study show that proliferative and degenerative characteristics are not uniformily distributed in the thecal microvascular networks. Active 
angiogenesis, as indicated morphologically by the presence of both sprouting and non-sprouting capillaries (Macchiarelli et al., 1991, 1995; Augustin et al., 1995; Yamada et al., 1995; Risau, 1997; Macchiarelli, 2000), was more evident in the largest follicles and more active in the inner plexus than the outer plexus. Indeed, capillary proliferation appeared to be distributed first in the apical region and then in the equatorial or basal regions of inner capillary layers. A recent study on the microvascular network of bovine ovarian follicles indicated that von Willebrand factor- (vWF, a specific marker for endothelial damage) positive areas varied from region to region in the same follicles and between follicles according to health or atresia. In the theca interna, the vWF-positive area was significantly greater in advanced and late atretic follicles compared with healthy and early atretic follicles (Isobe et al., 2001). Angiogenesis was also accompanied by vasodilation (mostly evident in the inner capillary layer of the largest follicles), a functional adaptation to the impending ovulation and to the developing thecal endocrine function (Macchiarelli, 2000). These findings indicate that secretion, distribution and action of angiogenic factors vary in different locations of follicles during development. In the present study, numerous degenerative capillaries in medium plexuses were also observed. Degenerative capillaries were localized in numerous small vascular plexuses in the outer layer, whereas medium plexuses also showed degenerative capillaries in the inner layer. These data are in accordance with the observations in the present study that apoptosis is first apparent in the outer and subsequently in the inner thecal capillaries, and vWFpositive areas are most evident in the outer layer of the theca interna in healthy follicles (Isobe et al., 2001). These findings support the hypothesis that capillary degeneration is linked to endothelial programmed cell death and is maximal when ovarian follicles undergo atresia.

It has been suggested that heterogeneity in follicular vascularization may be important in determining the fate of the developing follicles (continued growth versus atresia; Bassett, 1943; Burr and Davies, 1951) and the maintenance of the follicular vasculature is important for follicular health (Mattioli et al., 2001). For example, atretic ovine follicles regenerate if placed in culture, indicating that their access to nutrients and gonadotrophins could be limited in vivo (Moor and Seamark, 1986). In addition, earlier studies indicate that atresia in bovine follicles starts in the follicular wall rather than in the oocyte (Kenney, 1973) and in early atretic follicles there is a consistent reduction in capillaries in the theca interna (Marion et al., 1968). Greenwald (1989) also reported decreased proliferation of thecal capillary endothelial cells and reduced thecal vascularity as early events during follicular atresia, an observation that appears consistent with the onset of atresia in bovine, ovine and pig follicles (Fricke et al., 1996, 1997;
Jablonka-Shariff et al., 1996; Reynolds and Redmer, 1998). The present study also shows the presence of many large avascular areas in the inner layer of the atretic medium and large follicles (oestradiol:progesterone ratios $<1.0$ ), indicating that thecal vascularity was markedly reduced in atretic follicles.

The causal mechanism of atresia has not been determined and it is not known why some granulosa and thecal cells degenerate faster than others (Lobel and Levy, 1968). Although a previous study has shown that in the stratum granulosum and theca interna atresia begins on one side of the follicle while the opposite side remains reasonably normal (Marion et al., 1968), the reason(s) for this region-specific phenomenon is not known. Recent studies indicate that vascular endothelial growth factor and fibroblast growth factor are major angiogenic factors responsible for vascular development in ovaries (Reynolds et al., 1992, 1994; Redmer and Reynolds, 1996; Jiang et al., 2000b, 2001b; Shimizu et al., 2002). Therefore, further studies on the secretion, regulation and topographical expression of these factors will provide important insight into the role and the control mechanism(s) involved in the regulation of angiogenesis during ovarian follicular development and atresia.

In summary, the current study has shown that (1) active angiogenesis increases during bovine ovarian follicular growth; it is first evident in the follicular apical region of the inner theca layer; and (2) capillary degeneration, a consequence of apoptosis of outer theca interna capillaries, occurs unevenly and first appears in the outer vascular layers of the follicles and is likely to be associated with the process of atresia.

The authors would like to thank the staff of the abattoir in Sendai City for supplying bovine ovaries. Thanks are also due to K. Kimura for his help in the collection and transportation of bovine ovaries and to T. Sato of the Electron Microscopy Laboratory, Graduate School of Agricultural Science in Tohoku University for his kind assistance and co-operation during SEM observations. This work was supported by grants from the Program for Promotion of Basic Research Activities for Innovative Biosciences and the 'Research for the Future' Program, the Japan Society for the Promotion of Science (JSPS-RFTF97L00904), the Italian National Council of Research (CNR) and the Canadian Institutes of Health Research (MOP-15691).

\section{References}

Augustin HG, Braun K, Telemenakis I, Modlich U and Kuhn W (1995) Ovarian angiogenesis. Phenotypic characterization of endothelial cells in a physiological model of blood vessel growth and regression American Journal of Pathology 147 339-351

Bassett DL (1943) The changes in the vascular pattern of the ovary of the albino rat during the estrous cycle American Journal of Anatomy 73 $251-278$

Bjersing L and Cajander S (1974) Ovulation and the mechanism of follicle rupture. VI. Ultrastructure of theca interna and the inner vascular network surrounding rabbit graafian follicles prior to induced ovulation Cell and Tissue Research 153 31-44 
Burr JHJ and Davies JI (1951) The vascular system of the rabbit ovary and its relationship to ovulation The Anatomical Record 111 273-297

Cherney DD, Didio LJ and Motta P (1975) The development of rabbit ovarian follicles following copulation Fertility and Sterility 26 257-270

Ellinwood WE, Nett TM and Niswender GD (1978) Ovarian vasculature: structure and function. In The Vertebrate Ovary. Comparative Biology and Evolution pp 583-614 Ed. RE Jones. Plenum Press, New York

Espey LL (1980) Ovulation as an inflammatory reaction - a hypothesis Biology of Reproduction 22 73-106

Ferrara N, Carver-Moore K, Chen H, Dowd M, Lu L, O'Shea KS, PowellBraxton L, Hillan KJ and Moore MW (1996) Heterozygous embryonic lethality induced by targeted inactivation of the VEGF gene Nature $\mathbf{3 8 0}$ 439-442

Forsman AD and McCormack JT (1992) Microcorrosion casts of hamster luteal and follicular vasculature throughout the estrous cycle The Anatomical Record 233 515-520

Fricke PM, Ford JJ, Reynolds LP and Redmer DA (1996) Growth and cellular proliferation of antral follicles throughout the follicular phase of the estrous cycle in Meishan gilts Biology of Reproduction 54 879-887

Fricke PM, al-Hassan MJ, Roberts AJ, Reynolds LP, Redmer DA and Ford JJ (1997) Effect of gonadotropin treatment on size, number, and cell proliferation of antral follicles in cows Domestic Animal Endocrinology 14 171-180

Garrett WM and Guthrie HD (1996) Expression of androgen receptors and steroidogenic enzymes in relation to follicular growth and atresia following ovulation in pigs Biology of Reproduction 55 949-955

Ginther OJ and Del Campo CH (1974) Vascular anatomy of the uterus and ovaries and the unilateral luteolytic effect of the uterus: cattle American Journal of Veterinary Research 35 193-203

Greenwald GS (1989) Temporal and topographic changes in DNA synthesis after induced follicular atresia Biology of Reproduction 41 175-181

Greenwald GS and Terranova PF (1988) Follicular selection and its control. In The Physiology of Reproduction pp 387-445 Ed. G Neill. Raven Press, New York

Guthrie HD, Bolt DJ and Cooper BS (1993) Changes in follicular estradiol17 beta, progesterone and inhibin immunoactivity in healthy and atretic follicles during preovulatory maturation in the pig Domestic Animal Endocrinology 10 127-140

Guthrie HD, Cooper BS, Welch GR, Zakaria AD and Johnson LA (1995) Atresia in follicles grown after ovulation in the pig: measurement of increased apoptosis in granulosa cells and reduced follicular fluid estradiol-17 beta Biology of Reproduction 52 920-927

Isobe N, Kawai H, Yoshimura Y and Nakao T (2001) Changes in the localization of immuroreactive von Willebrand factor in microvascular network of bovine ovarian follicles during atresia Animal Science Journal 72 473-482

Jablonka-Shariff A, Reynolds LP and Redmer DA (1996) Effects of gonadotropin treatment and withdrawal on follicular growth, cell proliferation, and atresia in ewes Biology of Reproduction 55 693702

Jiang JY, Umezu M and Sato E (2000a) Improvement of follicular development rather than gonadotrophin secretion by thyroxine treatment in infertile immature hypothyroid $r d w$ rats Journal of Reproduction and Fertility 119 193-199

Jiang JY, Umezu M and Sato E (2000b) Thyroxine treatment promoted ovarian follicular angiogenesis by regulating gene expression of angiogenic factors in immature hypothyroid $r d w$ rats Biology of Reproduction $\mathbf{6 2}$ Supplement 1229

Jiang JY, Imai Y, Umezu M and Sato E (2001a) Characteristics of infertility in female hypothyroid (hyt) mice Reproduction 122 695-700

Jiang JY, Umezu M, Macchiarelli G and Sato E (2001b) Ovarian microvasculature and angiogenic regulation in follicular development and atresia. In Reproductive Biotechnology pp 73-80 Ed. N Manabe. Hokuto Shobo, Kyoto

Jiang JY, Macchiarelli G, Miyabayashi K and Sato E (2002) Follicular microvasculature in the porcine ovary Cell and Tissue Research $\mathbf{3 1 0}$ 93-101

Kanzaki H, Okamura H, Okuda Y, Takenaka A, Morimoto K and Nishimura T (1982) Scanning electron microscopic study of rabbit ovarian follicle microvasculature using resin injection-corrosion casts Journal of Anatomy 134 697-704

Kardon RH and Kessel RG (1979) SEM studies on vascular casts of the rat ovary Scanning Electron Microscopy 3 743-750

Kenney RM (1973) Histoenzymologic evaluation of metabolic patterns in bovine follicular oocytes American Journal of Veterinary Research $\mathbf{3 4}$ 893-896

Kikuta A, Macchiarelli G and Murakami T (1991) Microvasculature of the ovary. In Ultrastructure of the Ovary pp 239-254 Ed. PM Motta. Kluwer Academic Publishers, Boston

Kitai H, Yoshimura Y, Wright KH, Santulli R and Wallach EE (1985) Microvasculature of preovulatory follicles: comparison of in situ and in vitro perfused rabbit ovaries following stimulation of ovulation American Journal of Obstetrics and Gynecology 152 889-895

Lametschwandtner A, Lametschwandtner U and Weiger T (1990) Scanning electron microscopy of vascular corrosion casts - technique and applications: updated review Scanning Microscopy 4 889-941

Lamond DR and Drost M (1974) Blood supply to the bovine ovary Journal of Animal Science 38 106-112

Lang RA and Bishop JM (1993) Macrophages are required for cell death and tissue remodeling in the developing mouse eye Cell 74 453-462

Lang R, Lustig M, Francois F, Sellinger M and Plesken H (1994) Apoptosis during macrophage-dependent ocular tissue remodelling Development 120 3395-3403

Lobel BL and Levy E (1968) Enzymic correlates of development, secretory function and regression of follicles and corpora lutea in the bovine ovary Acta Endocrinologica (Copenhagen) Supplement 132 135-163

Macchiarelli G (2000) The microvasculature of the ovary: A review by SEM of vascular corrosion casts Journal of Reproduction and Development 46 207-225

Macchiarelli G, Nottola SA, Vizza E, Kikuta A, Murakami T and Motta PM (1991) Ovarian microvasculature in normal and hCG stimulated rabbits. A study of vascular corrosion casts with particular regard to the interstitium Journal of Submicroscopic Cytology and Pathology 23 391-395

Macchiarelli G, Vizza E, Nottola SA, Familiari G and Motta PM (1992) Cellular and microvascular changes of the ovarian follicle during folliculogenesis: a scanning electron microscopic study Archives of Histology and Cytology 55 Supplement 191-204

Macchiarelli G, Nottola SA, Vizza E, Familiari G, Kikuta A, Murakami T and Motta PM (1993) Microvasculature of growing and atretic follicles in the rabbit ovary: a SEM study of corrosion casts Archives of Histology and Cytology 56 1-12

Macchiarelli G, Nottola SA, Vizza E, Correr S and Motta PM (1995) Changes of ovarian microvasculature in hCG stimulated rabbits. A scanning electron microscopic study of corrosion casts Italian Journal of Anatomy and Embryology 100 Supplement 1 469-477

Macchiarelli G, Nottola SA, Picucci K, Stallone T and Motta PM (1998) The microvasculature of the corpus luteum in pregnant rabbit. A scanning electron microscopy study of corrosion casts Italian Journal of Anatomy and Embryology 103 191-202

Marion GB, Gier HT and Choudary JB (1968) Micromorphology of the bovine ovarian follicular system Journal of Animal Science 27 451-465

Mattioli M, Barboni B, Turriani M, Galeati G, Zannoni A, Castellani G, Berardinelli P and Scapolo PA (2001) Follicle activation involves vascular endothelial growth factor production and increased blood vessel extension Biology of Reproduction 65 1014-1019

Meeson A, Palmer M, Calfon M and Lang R (1996) A relationship between apoptosis and flow during programmed capillary regression is revealed by vital analysis Development 122 3929-3938

Meeson AP, Argilla M, Ko K, Witte L and Lang RA (1999) VEGF deprivationinduced apoptosis is a component of programmed capillary regression Development 126 1407-1415

Meyer GT (1991) Ultrastructural dynamics during corpus luteum development and growth. In Ultrastructure of the Ovary pp 161-176 Ed. PM Motta. Kluwer Academic Publishers, Boston

Miodonski AJ and Litwin JA (1999) Microvascular architecture of the human urinary bladder wall: a corrosion casting study The Anatomic Record $\mathbf{2 5 4}$ 375-381 
Moor RM and Seamark RF (1986) Cell signaling, permeability, and microvasculatory changes during antral follicle development in mammals Journal of Dairy Science 69 927-943

Murakami T (1971) Application of the scanning electron microscope to the study of fine distribution of the blood vessels Archivum Histologicum Japonicum 32 445-454

Murakami T, Ikebuchi Y, Ohtsuka A, Kikuta A, Taguchi T and Ohtani O (1988) The blood vascular wreath of rat ovarian follicle, with special reference to its changes in ovulation and luteinization: a scanning electron microscopic study of corrosion casts Archives of Histology and Cytology 51 299-313

Murdoch WJ and Cavender JL (1989) Effect of indomethacin on the vascular architecture of preovulatory ovine follicles: possible implication in the luteinized unruptured follicle syndrome Fertility and Sterility 51 153155

Nottola SA, Macchiarelli G and Motta PM (1997) The angioarchitecture of estrous, pseudopregnant and pregnant rabbit ovary as seen by scanning electron microscopy of vascular corrosion casts Cell and Tissue Research 288 353-363

Redmer DA and Reynolds LP (1996) Angiogenesis in the ovary Reviews of Reproduction 1 182-192

Reynolds LP and Redmer DA (1998) Expression of the angiogenic factors, basic fibroblast growth factor and vascular endothelial growth factor, in the ovary Journal of Animal Science 76 1671-1681

Reynolds LP, Killilea SD and Redmer DA (1992) Angiogenesis in the female reproductive system FASEB Journal 6 886-892

Reynolds LP, Grazul-Bilska AT, Killilea SD and Redmer DA (1994) Mitogenic factors of corpora lutea Progress in Growth Factor Research 5 159-175

Risau W (1997) Mechanisms of angiogenesis Nature 386 671-674

Roth Z, Meidan R, Shaham-Albalancy A, Braw-Tal R and Wolfenson D (2001) Delayed effect of heat stress on steroid production in mediumsized and preovulatory bovine follicles Reproduction 121 745751
Shimizu T, Jiang JY, Sasada H and Sato E (2002) Changes of mRNA expression of angiogenic factors and related receptors during follicular development in gilts Biology of Reproduction 67 1846-1852

Shimoda K, Sato E, Tanaka T and Toyoda Y (1993) Morphological differentiation of the microvasculature during follicular development, ovulation and luteinization of mouse ovaries Development, Growth and Differentiation 35 431-437

Spanel-Borowski K, Amselgruber W and Sinowatz F (1987) Capillary sprouts in ovaries of immature superstimulated golden hamsters: a SEM study of microcorrosion casts Anatomy and Embryology (Berlin) 176 387-391

Takada S, Shimada T, Nakamura M, Mori H and Kigawa T (1987) Vascular pattern of the mammalian ovary with special reference to the threedimensional architecture of the spiral artery Archivum Histologicum Japonicum 50 407-418

Yamada H, Yamada E, Hackett SF, Ozaki H, Okamoto $\mathbf{N}$ and Campochiaro PA (1999) Hyperoxia causes decreased expression of vascular endothelial growth factor and endothelial cell apoptosis in adult retina Journal of Cellular Physiology 179 149-156

Yamada O, Abe M, Takehana K, Iwasa K, Hiraga T and Hiratsuka T (1994) Microvasculature of mature bovine follicles and its changes with ovulation Journal of Reproduction and Development 40 307-315

Yamada O, Abe M, Takehana K, Hiraga T, Iwasa K and Hiratsuka T (1995) Microvascular changes during the development of follicles in bovine ovaries: a study of corrosion casts by scanning electron microscopy Archives of Histology and Cytology 58 567-574

Received 4 September 2002.

First decision 7 November 2002.

Revised manuscript received 8 November 2002.

Accepted 11 November 2002. 